AUTORES

Daniel Marcon ${ }^{1}$

Amândio Graça ${ }^{2}$

Juarez Vieira do Nascimento ${ }^{3}$

${ }^{1}$ Curso de Educação Física, Universidade de Caxias do Sul (UCS), Brasil

${ }^{2} \mathrm{CIFI}^{2} \mathrm{D}$, Faculdade de Desporto Universidade do Porto, Portugal

${ }^{3}$ Centro de Desportos, Universidade Federal de Santa Catarina (UFSC), Brasil

https://doi.org/10.5628/rpcd.11.03.127
Considerações teóricas

\section{sobre o conhecimento}

pedagógico do conteúdo

na formação inicial

em Educação Física.

\section{PALAVRAS CHAVE:}

Base de conhecimentos para o ensino.

Formação de professores. Conhecimentos

docentes. Ensino superior.

\title{
RESUMO
}

Este ensaio pretende contribuir para uma reflexão teórica sobre a construção do conhecimento pedagógico do conteúdo (CPC) e o papel de charneira que desempenha no processo de gestão da base de conhecimentos para o ensino nas práticas pedagógicas da formação inicial em Educação Física. Integrante dessa base de conhecimentos, juntamente com os conhecimentos dos alunos, do conteúdo, pedagógico geral e do contexto, o CPC tem por incumbência transformar conhecimentos do conteúdo em conhecimentos ensináveis. Daí, é desafio da formação inicial desenvolver o CPC dos estudantes-professores para que, por meio de experiências de prática pedagógica relevantes e adequadas, aprendam a gerir a base de conhecimentos e a ajustar decisões e intervenções às particularidades das situações de ensino e aprendizagem. Nesse processo, o CPC recebe informações das situações de ensino e aprendizagem; convoca conhecimentos na base de conhecimentos; faz interagir conhecimentos e informações; estabelece estratégias de ação; intervém na situação-problema; avalia resultados; e arquiva os novos conhecimentos na base de conhecimentos. Como resultado dessa intervenção do $\mathrm{CPC}$ num ambiente de prática enriquecedor, toda a base de conhecimentos é reconstruída e aperfeiçoada, o que favorece a construção do próprio CPC e o alcance dos objetivos da formação dos futuros professores de Educação Física. 


\title{
Theoretical considerations
}

\author{
on the pedagogical knowledge \\ of the content of the initial training \\ in Physical Education
}

\section{ABSTRACT}

This essay intends to advance the theoretical understanding about the construction of pedagogical content knowledge (PCK), and about the critical role it plays in managing the knowledge base for teaching throughout field experiences in PE teacher education. The knowledge base encompasses knowledge; concepts and dispositions built at different moments, contexts and experiences of prospective-teachers through their personal, academic and professional pathways. It is composed of student knowledge, content knowledge, pedagogical knowledge, context knowledge, and PCK, which works to transform content knowledge into teachable knowledge. Thus, the challenge is to develop prospective-teachers' PCK through relevant, nurturer teaching experiences, enabling them to learn how to manage the knowledge base and to adjust decision making to the particularities of learning situations. In this process, PCK (1) is informed by teaching and learning situations (2) convenes knowledge in the knowledge base, (3) puts in interaction knowledge and information; (4) establishes strategies for action, (5) intervenes in the problem situation, (6) evaluates results, and (7) stores new knowledge within the knowledge base. The PCK intervention in a nurturer environment acts to rebuild the knowledge base for teaching, which in turn will improve PCK and the achievement of the educational goals for the training of prospective PE teachers.

\section{KEY WORDS:}

Knowledge base for teaching. Teacher training. Teacher's knowledge.

Higher education. 
Nos últimos anos, o tema referente à construção do conhecimento pedagógico do conteúdo e à implementação de seus pressupostos na Educação Básica e na Superior tem sido disseminado e adaptado às diferentes áreas do conhecimento, bem como aos próprios contextos de formação inicial e continuada de professores $(6,10,11,15,16,22,25,27,31,37)$.

A literatura tem revelado que os professores utilizam suas inúmeras experiências de vida, sejam elas relacionadas aos contextos pessoal ou social, sejam elas relacionadas aos contextos acadêmico ou profissional, para sustentar a estruturação de suas concepções e a construção de seus conhecimentos, saberes, habilidades e competências docentes $44,12,13,20$ 42). Tem-se verificado que os professores utilizam esses conhecimentos, saberes, habilidades e competências docentes para adquirir e aperfeiçoar sua capacidade de transformar seus próprios conhecimentos sobre o conteúdo em prol da aprendizagem de seus alunos $(6,12,15,18,22$, $35,37,38)$. No entanto, a tentativa de explicar como interagem e se processam esses elementos durante a vida acadêmica dos futuros professores, e como convergem para a construção do con hecimento pedagógico do conteúdo, ainda revela lacunas que suscitam ques-

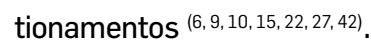

Nesse cenário, o presente ensaio se propõe a contribuir para uma reflexão teórica a respeito quer da construção do conhecimento pedagógico do conteúdo dos futuros professores; quer do papel de charneira desempenhado pelo conhecimento pedagógico do conteúdo na gestão dos ingredientes da base de conhecimentos durante as práticas pedagógicas na formação inicial em Educação Física.

Para dar conta desses objetivos, e visando a alcançar a profundidade necessária para a análise, optou-se por estruturar o texto em quatro pontos que analisam facetas complementares da relação entre a base de conhecimentos para o ensino e o conhecimento pedagógico do conteúdo de futuros professores de Educação Física. Essas facetas estão relacionadas à apresentação (1) da base de conhecimentos para o ensino; (2) do conhecimento pedagógico do conteúdo; (3) de algumas das possíveis relações entre a base de conhecimentos e o conhecimento pedagógico do conteúdo; e (4) de algumas das possíveis relações entre a base de conhecimentos e o processo de ensino e aprendizagem.

\section{BASE DE CONHECIMENTOS PARA O ENSINO}

A base de conhecimentos para o ensino, na proposta original de Shulman ${ }^{(38)}$, se refere a um corpo de conhecimentos, concepções e disposições construído em diferentes momentos, contextos e experiências vividas pelo professor ao longo de sua trajetória pessoal, escolar, acadêmica e profissional. De acordo com o autor ${ }^{(38)}$, a base de conhecimentos é formada por sete diferentes conhecimentos, nomeadamente: do conteúdo; pedagógico geral; do currículo, dos estudantes e de suas características; do contexto educacional; dos 
fins, propósitos e valores educacionais; e pedagógico do conteúdo.

$\mathrm{Na}$ sua esteira, diferentes estudiosos procuraram não apenas contribuir para a elucidação da base de conhecimentos dos futuros professores, como também e, fundamentalmente, viabilizar a adaptação e a implementação dessa proposta pelos programas de formação inicial de professores tanto de Educação Física quanto de outras áreas $(9,10,15,18,29,32)$.

De maneira geral, um dos papéis fundamentais da base de conhecimentos para a formação e para a atuação dos professores de Educação Física é o de congregar conhecimentos necessários para a docência, os quais poderão ser requisitados em diferentes momentos da prática pedagógica do futuro professor.

Estudos relacionados à formação inicial de professores evidenciam que, mesmo conhecendo um assunto, ao mesmo tempo e a partir das mesmas estratégias, a forma como cada estudante-professor interpreta, assimila e constrói seus novos conhecimentos é diferente ${ }^{(12,26,30,41)}$. Tal diferenciação ocorre em virtude da relação dinâmica de todos os elementos formativos aos quais o estudante-professor esteve e está submetido ${ }^{24,40,42)}$, quer sejam experiências de vida prévias, escolares, esportivas, acadêmicas (no contexto universitário) e profissionais ${ }^{(5,14,19,33,36,42,46)}$. Conforme ensina Graça ${ }^{(15)}$, "as novas aprendizagens constroem-se com base no conhecimento anterior que o sujeito possui. Esse conhecimento (onde se inclui também as concepções ingênuas e erros conceituais) dita a sorte da nova informação" (p.51).

Como resultado de seus estudos sobre a formação inicial de professores, Meirieu (26) obteve algumas "evidências pedagógicas" que reforçam esses aspectos:

Cada aluno aprende de uma maneira que the é própria [...]. Cada um dispõe de recursos próprios nos quais pode apoiar-se para the permitir superar-se [...]. A identificação por parte de cada uma das suas aquisições é uma condição de acesso à sua autonomia [...]. A interação entre colegas é uma riqueza inestimável que permite a cada um apropriar-se de seu próprio saber e incorporar progressivamente o ponto de vista de outro para desenvolver-se" (p.31).

Nesse mesmo sentido, Ennis ${ }^{(12)}$ explica que a pesquisa em organização do conhecimento em Educação Física [...] identificou tanto similaridades entre estruturas do conhecimento de professores que têm treinamento e experiência comuns, quanto diferenças nas formas como professores conceitualizam e usam a base de conhecimentos. Isso sugere que alguns componentes da organização do conhecimento são únicos para o professor individual. Cada professor entende o conhecimento baseado em experiências anteriores" (p.170). (tradução nossa).

Além disso, os conhecimentos relacionados ao conteúdo não estão prontos para serem diretamente aplicados pelo estudante-professor nas situações de ensino e aprendizagem, 
seja naquelas onde atua como professor-estudante, seja naquelas que são apenas objeto de análise e reflexão ${ }^{(3,5,15)}$. Por terem sido elaborados a partir das vivências e experiências próprias de cada estudante-professor, esses conhecimentos são individuais e só têm significado e fazem sentido para ele ${ }^{(40)}$. Ditos conhecimentos são úteis somente para o próprio estudante-professor e, da forma como foram concebidos, não servem aos outros e não contribuem para o aprendizado dos alunos $(5,16,19,23,27)$.

Muitos desses conhecimentos, apesar de serem dele, do estudante-professor, não são para ele, pois foram construídos na formação inicial docente com a principal finalidade de

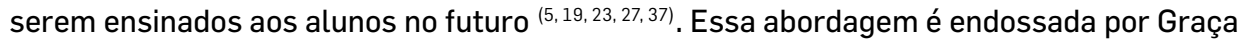
(15), ao argumentar que "ao professor exige-se-lhe [...] que saiba representar a matéria para os outros; não the basta saber para si [...]. É este espaço do saber para os outros que configura a especificidade do conhecimento do professor" (p.81).

\section{CONHECIMENTO PEDAGÓGICO dO CONTEÚDO}

Além de a estrutura da base de conhecimentos e de a disposição dos conhecimentos na sua essência serem individuais, a gestão e as estratégias utilizadas para aplicá-los nas situações de ensino e aprendizagem também são particulares de cada estudante-professor, uma vez que estarão condicionadas às suas concepções e experiências prévias ${ }^{(3,14,30,41,42)}$.

Para que os conhecimentos relacionados ao conteúdo possam ser ensinados, além de necessitarem estar bem-estruturados, terão de passar por um tratamento, sob o ponto de vista do seu ensino, por uma transformação à luz dos conhecimentos pedagógicos e dos demais conhecimentos concernentes à situação de ensino e aprendizagem $(5,8,16,27,28,37)$ Como esclarece Amade-Escot ${ }^{(3)}$, "o conhecimento a ser ensinado e o conhecimento realmente ensinado passam por complexos processos de transformação, em vários estágios de seleção e ensino, o que distingue essencialmente este conhecimento das suas origens no conhecimento acadêmico" (p.87). (tradução nossa).

Vários autores compartilham dessa visão, ressaltando a necessidade de os programas de formação inicial em Educação Física implementarem mecanismos pedagógicos precursores (1) da conscientização dos estudantes-professores sobre a sua relação com seus próprios conhecimentos; (2) do esclarecimento quanto a justificativas e objetivos para a construção de cada um dos seus conhecimentos e, principalmente; (3) da construção do conhecimento pedagógico do conteúdo, por meio da gestão de todos esses conhecimentos em situações reais de ensino e aprendizagem ${ }^{3,9,17,19,22,27,29,39,43)}$.

Nessa gestão, o conhecimento pedagógico do conteúdo comanda um processo envolvendo os conhecimentos dos alunos, do conteúdo para ensinar, pedagógico geral e do 
contexto, com o fito de submeter o conhecimento do conteúdo a ser ensinado a uma transformação ${ }^{(38)}$, a uma tradução ${ }^{(10)}$ que o converta em conhecimento ensinável e, consequentemente, útil aos alunos $(3,5,10,19,28,37,38)$.

Esse configura o próprio âmago do conhecimento pedagógico do conteúdo, pois é aquele que o estudante-professor utiliza para, a partir dos seus objetivos, da realidade dos alunos e das características do contexto de ensino e aprendizagem, convocar, gerir e fazer interagir os conhecimentos da base de conhecimentos para o ensino visando à adaptação, à transformação e à implementação do conhecimento do conteúdo a ser ensinado, de modo a torná-lo compreensível e ensinável aos alunos.

Ao fim desse processo, o conhecimento do conteúdo dele, do estudante-professor, passa a ser um conhecimento para os alunos, pois adquire uma nova estrutura e um novo caráter. Além de distingui-lo do conhecimento puramente acadêmico ${ }^{(3)}$, esse pode ser considerado, inclusive, um novo conhecimento sobre o mesmo assunto ${ }^{(27,41)}$. É justamente esse novo conhecimento aquele que pode ser ensinado, e não o primeiro.

\section{RELAÇÕES ENTRE BASE DE CONHECIMENTOS E CONHECIMENTO PEDAGÓGICO DO CONTEÚDO}

A maneira como os professores gerem seus conhecimentos, em diferentes situações de ensino e aprendizagem, constituiu objeto de estudo de vários autores ${ }^{(3,4,7,10,15,21,26,34,38,45)}$. Dentre os estudos destacam-se aqueles desenvolvidos por Shulman ${ }^{(38)}$, Rovegno ${ }^{(34)}$ e Meirieu ${ }^{(26)}$, os quais, apesar de origens e percursos distintos, vislumbram objetivos semelhantes.

Shulman ${ }^{(38)}$ denominou "processo de raciocínio e ação pedagógica" a sequência de eventos levada a cabo pelo professor na sua atuação docente, cujos integrantes são as etapas de: compreensão do assunto a ser ensinado; transformação do conhecimento do conteúdo do professor em conhecimento para o aluno; instrução ou implementação das estratégias planejadas; avaliação dos resultados obtidos; reflexão a respeito desses resultados; e construção de uma nova compreensão a respeito do assunto. 0 autor ressalta que, embora apresentadas em sequência, essas etapas podem tanto ser utilizadas nessa ordem quanto de maneira alternada, dependendo das características dos alunos e do contexto, da estrutura da base de conhecimentos do professor e dos objetivos a serem alcançados com a prática pedagógica ${ }^{(38)}$.

De acordo com pesquisas realizadas por Rovegno ${ }^{(34)}$, nas práticas pedagógicas em Educação Física, os professores podem gerir seus conhecimentos seguindo (a) um modelo linear, hierárquico e rígido, onde o conteúdo é dividido em unidades descontextualizadas e apresentadas separadamente; (b) um modelo espiral, menos linear e relativamente flexível, no qual os exercícios tornam-se progressivamente mais complexos e interligados; ou (c) um modelo de matriz centrado numa visão holística do conhecimento, que busca, nas suas origens, elementos capazes de relacioná-lo com os demais conhecimentos, com os significados que têm 
para os alunos e com os objetivos da situação de ensino e aprendizagem ${ }^{(3,4,34)}$.

Meirieu ${ }^{(26)}$, por outra parte, analisa essa temática a partir de três níveis de transferência. No primeiro nível, a "transferência remete à possibilidade de utilizar um conhecimento, uma competência ou um domínio de uma situação para outra" (p.217), pois tem como objetivo identificar semelhanças entre duas situações pedagógicas, "descontextualizar" o conhecimento construído em uma delas e tentar "recontextualizá-lo" na outra; abordar especificamente a questão da utilização futura de um conhecimento construído agora; e incitar, a cada novo dilema ou situação-problema, a busca de estratégias exitosas utilizadas em situações de ensino e aprendizagem anteriores.

No segundo nível, a "transferência remete à possibilidade de 'criar pontes' entre disciplinas diferentes, entre as situações de formação e de trabalho, entre saberes escolares e a vida pessoal do sujeito" (p.217) ${ }^{(26)}$. Essas pontes, segundo Meirieu ${ }^{(26)}$, supõem

uma verdadeira reconstrução de esquemas de ação em função daquilo que já foi aprendido e de elementos novos que são descobertos, em função também do ambiente particular no qual se encontra agora, de seus códigos sociais e de seus hábitos, da história específica de um sujeito que encontra outras histórias e deve levá-las em conta para incorporar aquilo que ele era e o que já sabia em uma situação social nova (p.217).

No terceiro nível, "a transferência remete à própria possibilidade de incorporar conhecimentos 'mortos' em uma dinâmica pessoal e de se construir como sujeito de seus próprios atos" (p.217) ${ }^{(26)}$, agindo consciente e intencionalmente para gerir e transferir, de uma situação a outra, os integrantes da base de conhecimentos, para onde retornarão sob a forma de novos conhecimentos $(7,21,45)$.

As reflexões desses autores, bem como os resultados das pesquisas realizadas por outros como Altet ${ }^{(2)}$, Behets ${ }^{(4)}$, Cochran, DeRuiter e King ${ }^{(9)}$, Hegarty ${ }^{(21)}$, Jenkins e Veal ${ }^{(22)}$ e Park e Oliver ${ }^{(29)}$, podem ser indicadoras de algumas das responsabilidades assumidas pelo próprio conhecimento pedagógico do conteúdo, justamente por darem pistas da maneira como gere a base de conhecimentos em diferentes situações de ensino e aprendizagem. A confluência de todas essas perspectivas leva-nos a conceber o conhecimento pedagógico do conteúdo como o responsável por (1) receber as informações extraídas das situações de ensino e aprendizagem por meio das reflexões; (2) convocar os conhecimentos na base de conhecimentos; (3) fazer interagir esses conhecimentos e essas informações; (4) estabelecer estratégias de ação; (5) intervir na situação-problema; (6) avaliar seus resultados; e (7) arquivar os novos conhecimentos na base de conhecimentos. Esse sistema é retroalimentado pelas informações advindas do próprio contexto, as quais, por terem sido geradas a partir da própria intervenção na prática, mantêm o sistema em funcionamento $(2,4,9,21,22,29)$.

A análise de cada uma dessas tarefas permite-nos sustentar que o conhecimento pedagógico do conteúdo não é apenas um dos integrantes da base de conhecimentos para o en- 
sino, mas é aquele com autonomia para transitar tanto no seu interior quanto fora dela, e é o responsável pelas "transferências", pelas ligações entre a base e a prática pedagógica (21, 22, 29). Como esclarece Mizukami ${ }^{(27)}$, o conhecimento pedagógico do conteúdo se refere a um novo tipo de conhecimento, que é construído constantemente pelo professor ao ensinar a matéria e que é enriquecido e melhorado quando se amalgamam os outros tipos de conhecimentos explicitados na base [...]. Inclui compreensão do que significa ensinar um tópico de uma disciplina específica assim como os princípios e técnicas que são necessários para tal ensino (p.5).

Esse conjunto de tarefas, e, principalmente a sua inter-relação, tem potencial para definir não apenas a estrutura do conhecimento pedagógico do conteúdo do futuro professor de Educação Física, mas também e fundamentalmente o seu perfil docente, oferecendo elementos para vislumbrar, inclusive, como será no desempenho da profissão.

\section{RELAÇÕES ENTRE BASE DE CONHECIMENTOS E PROCESSO DE ENSINO E APRENDIZAGEM}

Como observado na análise anterior, ter uma vasta base de conhecimentos, repleta de conhecimentos relacionados, por exemplo, a conteúdos de diferentes áreas, só se justifica à ação pedagógica quando, por intermédio do conhecimento pedagógico do conteúdo, todos estiverem à disposição das outras pessoas, quando forem utilizados para construir os conhecimentos dos alunos e quando contribuírem para seu aprendizado ${ }^{(16,19,37,38)}$.

Além de repercutir na estrutura organizacional, esses processos de gestão e de transformação também determinarão o tempo de permanência de cada um dos conhecimentos em posição de destaque dentro da base de conhecimentos. Esse destaque dependerá, basicamente, da frequência com que serão convocados e da importância de cada conhecimento para o alcance dos objetivos e a solução dos dilemas nas situações de ensino e aprendizagem. Se determinado conhecimento, apesar de ter sido relevante anteriormente, não mais for convocado pelo conhecimento pedagógico do conteúdo, ele passa a ser substituído por outros que, em função das características do contexto de ensino e aprendizagem, ofereçam maiores possibilidades de solução dos dilemas e das situações-problema ${ }^{(21,41)}$.

À medida que esse conhecimento for novamente convocado, ele será utilizado na situação de ensino e aprendizagem e, por meio das reflexões relacionadas à prática pedagógica, será reformulado e fortalecido pelas novas experiências, assumindo um novo status na base de conhecimentos. Esse caracteriza o ciclo dinâmico de convocação e de gestão da base de conhecimentos pelo conhecimento pedagógico do conteúdo. Se for convocado frequentemente, começam a ser consolidadas as concepções, as crenças e a confiança do estudante-professor quanto à eficiência desse conhecimento na solução dos dilemas surgidos nas situações 
de ensino e aprendizagem ${ }^{(1,6,12,14,16,19,21,41,44)}$. Graça ${ }^{(15)}$ contribui para o esclarecimento da dinâmica dessa relação ao destacar que a aprendizagem

envolve uma interação entre uma nova concepção e outra já existente, cujo resultado depende da natureza desta interação. Se houver possibilidade de conciliação entre as concepções, a aprendizagem processa-se sem dificuldade. Se tal não for o caso, a aprendizagem exigirá a reestruturação da concepção existente ou mesmo a mudança para uma nova concepção (p.51).

Dessa forma, os conhecimentos aos quais o estudante-professor terá acesso mais direto são aqueles que se destacam em sua base de conhecimentos, possuem relevo por terem sido utilizados recentemente, ou por várias vezes, e auxiliaram na solução de dilemas em situações de ensino e aprendizagem anteriores ${ }^{(7,21)}$.

Vale ressaltar que as concepções e os conhecimentos dos estudantes-professores são constantemente revistos e reestruturados, em um fluxo contínuo de idas e vindas entre suas vivências e sua base de conhecimentos. Gerido pelo conhecimento pedagógico do conteúdo, esse processo se estenderá não só pelo período da formação inicial, mas ao longo de sua vida profissional ${ }^{(5,6,14)}$.

Quando determinados conhecimentos são convocados intencionalmente, contribuem para o alcance dos objetivos e respondem aos dilemas das situações de ensino e aprendizagem, então começa a ser observada a evolução da autocrítica e do discernimento do estudante-professor sobre a estrutura da sua base de conhecimentos e a capacidade do seu conhecimento pedagógico do conteúdo de adaptar à realidade os conhecimentos do conteúdo ${ }^{(3,12)}$. 0 futuro professor de Educação Física passa a demonstrar, assim, o amadurecimento do seu conhecimento pedagógico do conteúdo e da sua competência para, justamente, fazer com que seus próprios conhecimentos, relacionados ao conteúdo, se tornem ensináveis e compreensíveis por seus alunos ${ }^{(6,37)}$.

Tendo sido convocados reiteradas vezes, principalmente a partir de diferentes dilemas e em distintos contextos de ensino e aprendizagem, alguns conhecimentos começam a ganhar destaque na base de conhecimentos e a se tornar referência para a solução de outras situações-problema em práticas pedagógicas futuras ${ }^{(6,12,21,26,38,41)}$. Conforme esclarece Ennis ${ }^{(12)}$, quando os professores fazem um julgamento e o acham efetivo, é provável que incorporem a solução como parte de uma crença ou teoria informal [...]. Com o passar do tempo, teorias informais tornam-se altamente resistentes à mudança. Essas crenças rígidas influenciam as percepções dos professores sobre a utilidade de estratégias ou julgamentos alternativos, influenciando sua predisposição para considerar e usar nova informação (p.171). (tradução nossa).

Diante do exposto, observa-se que, ao desempenhar seu papel nas práticas pedagógicas, o conhecimento pedagógico do conteúdo, além de estreitar a relação entre a base de conhecimentos e o contexto de ensino e aprendizagem ${ }^{(9,21,22,29)}$, também é, ele próprio, 
delineado e reconstruído permanentemente. Como consequência, essa participação do conhecimento pedagógico do conteúdo nas práticas pedagógicas viabiliza a qualificação da formação docente dos estudantes-professores e o alcance de alguns dos objetivos dos programas de formação inicial de professores de Educação Física $(2,16,26,28,41,45)$.

\section{CONSIDERAÇÕES FINAIS}

Diante da sistematização das informações e das análises realizadas neste ensaio, verifica-se que tanto a base de conhecimentos para o ensino quanto o conhecimento pedagógico do conteúdo são diretamente influenciados pelas experiências de vida prévias, escolares, esportivas, acadêmicas (no contexto universitário) e profissionais às quais os estudantes-professores de Educação Física estiveram e estão submetidos. No que tange especificamente aos programas de formação inicial em Educação Física, ambientes propícios ao desenvolvimento do conhecimento pedagógico do conteúdo podem ser concebidos a partir da consideração, por exemplo, do local de realização das práticas pedagógicas, das características e do número de alunos, dos objetivos a serem alcançados e da estrutura e do tempo da aula.

A partir das proposições de vários autores, podem ser elaboradas algumas inferências a respeito das prováveis tarefas desempenhadas pelo conhecimento pedagógico do conteúdo nas práticas pedagógicas, como (1) receber, por meio de reflexões, as informações dos dilemas surgidos nas situações de ensino e aprendizagem; (2) convocar os conhecimentos na base de conhecimentos; (3) fazer interagir esses conhecimentos e essas informações; (4) estabelecer estratégias de ação; (5) intervir na situação-problema; (6) avaliar seus resultados; e (7) arquivar os novos conhecimentos na base de conhecimentos.

De modo geral, há o reconhecimento de que o conhecimento pedagógico do conteúdo é construído enquanto ele gere a relação entre a base de conhecimentos e a situação de ensino e aprendizagem, no sentido de tornar o conhecimento do conteúdo compreensível e ensinável aos alunos.

Entretanto, emergem alguns questionamentos relacionados (1) à maneira como o conhecimento pedagógico do conteúdo desempenha cada uma de suas diferentes tarefas e possibilita a interação entre a base de conhecimentos e as práticas pedagógicas; (2) aos elementos potencialmente influenciadores nesse processo; bem como (3) às estratégias possíveis de serem implementadas pelos programas de formação inicial de professores de Educação Física para viabilizar essas questões. Esses são, pois, alguns dos aspectos que justificam o aprofundamento dos estudos sobre o processo de construção do conhecimento pedagógico do conteúdo dos futuros professores de Educação Física e, por isso, têm potencial para nortear futuras reflexões sobre esse tema.

\section{AGRADECIMENTOS}

À Faculdade de Desporto da Universidade do Porto, na pessoa do Presidente do Conselho Directivo, Prof. Dr. Jorge Olímpio Bento, e à Universidade de Caxias do Sul. 
1. Alarcão I (1997). Contribuição da didática para a formação de professores: reflexões sobre o seu ensino. In: Pimenta SG (ed.), Didática e formação de professores: percursos e perspectivas no Brasil e em Portugal. São Paulo: Cortez: 159-190.

2. Altet M (2001). As competências do professor profissional: entre conhecimentos, esquemas de ação e adaptação, saber analisar. In: Paquay L, et al (eds.), Formando professores profissionais: Quais estratégias? Quais competências? Porto Alegre: Artmed, 23-35.

3. Amade-Escot C (2000). The contribution of two research programs on teaching content: "pedagogical content knowledge" and "didactics of physical education". Journal of Teaching in Physical Education, 20 (1): 78-101.

4. Behets $D$, Vergauwen $L$ (2006). Learning to teach in the field. In: Kirk D, Macdonald D, O'Sullivan M (eds), Handbook of physical education. London: Sage, 407-424.

5. Calderhead J (1988). The development of knowledge structures in learning to teach. In: Calderhead $J$ (ed) Teachers' professional learning. London/ Washington D.C. Falmer Press, 51-64.

6. Carter K (1990). Teachers knowledge and learning to teach. In: Sikula RHMHJ (ed), Handbook of research on teacher education. New York: MacMillan, 291-310.

7. Charlier É (2001). Formar professores profissionais para uma formação contínua articulada à prática. In: Paquay L, et al (eds.), Formando professores profissionais: Quais estratégias? Quais competências? Porto Alegre: Artmed, 85-102.

8. Chen W (2004). Learning the skill theme approach: salient and problematic aspects of pedagogical content knowledge. Education, 125 (2): 194-212.

9. Cochran KF, DeRuiter JA, King RA (1993). Pedagogical content knowing: an integrative model for teacher preparation. Journal of Teacher Education, 44 (4): 263-272.

10. Cochran KF, King RA, DeRuiter JA (1991). Pedagogical content knowledge: a tentative model for teacher preparation. Comunicação apresentada no Annual Meeting of the American Educational Research Association.Chicago.
11. Crum B (2000). Funções e competências dos professores de Educação Física: conseqüências para a formação inicial. Boletim da Sociedade Portuguesa de Educação Física, 1 (23): 61-76.

12. Ennis C (1994). Knowledge and beliefs underlying curricular expertise. Quest, 46 (2): 164-175.

13. Formosinho J (2001). A formação prática dos professores: da prática docente na instituição de formação à prática pedagógica nas escolas. Revista Portuguesa de Formação de Professores, 1: 37-54.

14. Gimeno Sacristán J (1995). Consciência e ação sobre a prática como libertação profissional dos professores, In: NóvoaA (ed.), Profissão professor. Porto: Porto Editora, 63-92.

15. Graça A (1997). O conhecimento pedagógico do conteúdo no ensino do basquetebol. Tese de doutoramento. Faculdade de Desporto da Universidade do Porto.

16. Graça A (2001). 0 conhecimento pedagógico do conteúdo: $O$ entendimento entre a pedagogia e a matéria. In: Gomes PB, Graça A (eds.), Educação física e desporto na escola: Novos desafios, diferentes soluções, Porto: FCDEF-UP: Porto, 107-120.

17. Griffin LL, Dodds PS, Rovegno I (1996). Pedagogical content knowledge for teachers: integrate everything you know to help students learn. Journal of Physical Education, Recreation and Dance, 67 (9): 58-61.

18. Grossman P (1990). The making of a teacher: Teacher knowledge and teacher education. New York: Teachers College Press.

19. Grossman P, Wilson S, Shulman L (1989). Teacher of substance: subject matter knowledge for teaching In: Reynolds M (ed.), Knowledge base for the beginning teacher. New York: Pergamon Press, 23-36.

20. Hayes S, Capel S, Katene W, Cook P (2008). An examination of knowledge prioritization in secondary physical education teacher education courses. Teaching and Teacher Education, 24 (2): 330-342.

21. Hegarty S (2000) Teaching as a knowledge-based activity. Oxford Review of Education 26, 451-465. DOI: 10.1080/3054980020001945.

22. Jenkins JM, Veal ML (2002). Preservice teachers' 
PCK development during peer coaching. Journal of Teaching in Physical Education, 22 (1): 49-68.

23. Lampert $M$ (1990). When the problem is not the question and the solution is not the answer: Mathematical knowing and teaching. American Educational Research Journal, 27 (1): 29-63.

24. Lima SM. Reali AM (2002). 0 papel da formação básica na aprendizagem profissional da docência (aprende-se a ensinar no curso de formação básica?). In: Mizukami MG, Reali AM (eds.), Formação de professores, práticas pedagógicas e escola. São Carlos: EdUFSCar, 217-236.

25. Marques AM (2000). Formação do profissional da educação (3ª ed.). Ijuí: Ed. da Unijuí.

26. Meirieu $P$ (2002). A pedagogia entre o dizer e o fazer: A coragem de começar. Porto Alegre: Artmed.

27. Mizukami MG (2004). Aprendizagem da docência: Algumas contribuições de L. S. Shulman. Revista Educação 29, 1-11.

28. Nascimento JV, Ramos, V, Marcon D, Saad MA, Collet $C$ (2009). Formação acadêmica e intervenção pedagógica nos esportes. Motriz, 15, 358-366.

29. Park S, Oliver JS (2008). Revisiting the conceptualisation of pedagogical content knowledge (PCK): PCK as a conceptual tool to understand teachers as professionals. Research in Science Education 38, 261284. DOI: 10.1007/s11165-007-9049-6.

30. Perdigão AL (2002). Concepções pessoais de futuros professores sobre os processos de aprendizagem e de ensino. In: Mizukami MG, Reali AM (eds.), Formação de professores, práticas pedagógicas e escola. São Carlos: EdUFSCar265-294.

31. Pimenta SG (2002). O estágio na formação de professores (5ª ed.). São Paulo: Cortez.

32. Ramos V, Graça A, Nascimento JV (2008). O conhecimento pedagógico do conteúdo: estrutura e implicações à formação em Educação Física. Revista Brasileira de Educação Física e Esporte 22, 161-171.

33. Reali AM, Mizukami MG (2002). Práticas profissionais, formação inicial e diversidade: análise de uma proposta de ensino e aprendizagem. In: Mizukami MG, Reali AM (eds.), Aprendizagem profissional da docên- cia: saberes, contextos e práticas. São Carlos: EdUFSCar, 119-138.

34. Rovegno I (1995). Theoretical perspectives on knowledge and learning and a student teacher's pedagogical knowledge of dividing and sequencing subject matter. Journal of Teaching in Physical Education, 14: 284-304.

35. Schincariol LM (2002). The types, sources, and perceived relevance of knowledge acquisition, and the enacted effects when teaching unfamiliar and familiar physical education content. Tese de doutoramento. The Ohio State University.

36. Schön DA (1995). Formar professores como profissionais reflexivos. In: Nóvoa A (ed.), Os professores e a sua formação. Lisboa: Dom Quixote, 77-91.

37. Segall A (2004). Revisiting pedagogical content knowledge: the pedagogy of content/the content of pedagogy. Teaching and Teacher Education, 20 (5): 489-504.

38. Shulman LS (1987). Knowledge and teaching: foundations of the new reform. Harvard Educational Review, 57 (1): 1-27.

39. Siedentop D (2002). Content knowledge for Physical Education. Journal of Teaching in Physical Education, 21 (4): 368-377.

40. Silva MLR (2008). Aplicação dos saberes na sala de aula: Desafios para o professor. Notandum XI, 46-52.

41. Solis Villa R (1984). Ideas intuitivas y aprendizaje de las ciencias. Investigación y experiencias didácticas 2, 83-89.

42. Tardif M, Raymond D(2000) Saberes, tempo e aprendizagem do trabalho no magistério. Educ. Soc. 21 209-244. DOI: 10.1590/S0101-73302000000400013. 43. Valli L, Rennert-Ariev P (2002). New standards and assessments? Curriculum transformation in teacher education. Journal of Curriculum Studies, 34 (2): 201-225.

44. Whipple CE (2002). Preservice teachers' views of content and pedagogical knowledge presented in the elementary component of a physical education teacher education program.Tese de doutoramento. The Ohio State University. 
45. Woods M, Goc Karp G, Escamilla E (2000). Preservice teachers learning about students and the teaching-learning process. Journal of Teaching in Physical Education, 20 (1): 15-39.

46. Zeichner KM (1994). Research on teacher thinking and different views of reflective practice in teaching and teacher education. In: Carlgren I, Handal G, Vaage S (eds.), Teachers' minds and actions: Research on teachers' thinking and practice. London/ Washington D.C, Falmer Press, 9-27 